\title{
Computational Determination of the Dirac-Theory Adjunctator
}

\author{
M. Dima \\ Institute for Physics and Nuclear Engineering, Atomistilor Street 407, P.O. Box MG-6, 76900 Bucharest, Romania \\ Correspondence should be addressed to M. Dima; modima@nipne.ro \\ Received 9 June 2013; Accepted 15 August 2013 \\ Academic Editor: Carlo Cattani \\ Copyright (C) 2013 M. Dima. This is an open access article distributed under the Creative Commons Attribution License, which \\ permits unrestricted use, distribution, and reproduction in any medium, provided the original work is properly cited. \\ A number of particle properties stem from the use of $\gamma^{0}$ as adjunctator (Bargmann-Pauli) in the Dirac theory (spin alignment, \\ Dirac current, etc.). The early motivations for accepting $\gamma^{0}$ as adjunctator were representation-dependent, mildly bearing relation \\ to the actual conditions forcing $\gamma^{0}$ as adjunctator. Representation-independent approaches to the physical predictions of the Dirac \\ equation are somewhat new, here presented as being the reasons for $\gamma^{0}$ as adjunctator of the Dirac theory, together with the essential \\ role of the latter in the physical aspects of the theory.
}

\section{Introduction}

The definition of the Dirac operators $(\beta, \vec{\alpha})$ as $\gamma^{0}(1, \vec{\gamma})$, satisfying $\left[\gamma^{\mu}, \gamma^{\nu}\right]_{+}=2 g^{\mu \nu} \cdot \mathbf{1}$, that is, a Clifford algebra $\mathscr{C} l_{1,3}(C)$, does not include any information on how adjoint operators are constructed. In representation-dependent form, as matrices, the adjoints are constructed as $\left(\gamma^{\mu^{\dagger}}\right)_{\alpha, \beta}$ $=\left(\gamma^{\mu}\right)_{\alpha, \beta}^{*}$; however, such representation-dependent solutions also include representation-specific artifacts, often nonphysical. The importance of presenting properties in representation-independent form, stemming from minimal information in the abstract definition of the foundations, thus cannot be emphasized enough.

The adjunctator (algebra element that constructs the adjoint) is present in a number of particle properties (current definition, spin alignment, etc.). In Dirac theory, this is the Bargmann-Pauli [1-3] adjunctator $\gamma^{0}$ and its unicity is crucial to knowing that the physical quantities used are well defined.

The initial demand in determining how the adjoint is constructed for $\left(\gamma^{0}, \vec{\gamma}\right)$ was from the Dirac-1928 equation itself as follows:

$$
\mathbf{E}=\mathbf{H}=\vec{\alpha} \cdot \overrightarrow{\mathbf{p}}+\beta m q_{q_{\psi}},
$$

where $\mathbf{E}$ is the energy, $\mathbf{H}$ the Hamiltonian, $(\beta, \vec{\alpha})$ Dirac's initial matrices, and $\psi$ the system's wave function. Since $\mathbf{E}=\mathbf{E}^{\dagger}$ it seemed natural to ask that $\mathbf{H}=\mathbf{H}^{\dagger}$. This is rather ad-hoc, all that the above relation has to offer being $\operatorname{Im}\left\{\left\langle\mathbf{H}^{\dagger}-\mathbf{H}\right\rangle_{\psi}\right\}=0$.
Leaving such "physics" arguments behind, the real aspects can be now explored. Little attention was given to this topic; however Pauli [3] did have interest in it at the time. His arguments though representation dependent (with one minor inaccuracy) offer the only explanation to date on this topic. The only representation-independent study $[4,5]$ is about the unicity of the adjunctator together with a pleiad of related studies $[6,7]$.

In the Dirac algebra (of the $\gamma^{\mu}$ matrices), the adjoint operators form another representation of the same algebra, as the arguments of Pauli [3] show the following:

$$
\left[\gamma^{\mu^{\dagger}}, \gamma^{\nu^{\dagger}}\right]_{-}=-\left[\gamma^{\mu}, \gamma^{\nu}\right]_{-}^{\dagger}=2 g^{\mu} \nu \cdot \mathbf{1} \Longrightarrow \gamma^{\mu^{\dagger}}=\mathbf{B} \gamma^{\mu} \mathbf{B}^{-1} .
$$

The two representations are thus related through a similarity transformation $\mathbf{B}$ termed adjunctator or hermitiser [1, 2], with the following properties:

$$
\begin{aligned}
\text { (i) } & \left(\gamma^{\mu \dagger}\right)^{\dagger}=\gamma^{\mu}=\mathbf{B}^{-1^{\dagger}} \gamma^{\mu} \mathbf{B}^{\dagger}=\left(\mathbf{B}^{-1^{\dagger}} \mathbf{B}\right) \gamma^{\mu}\left(\mathbf{B}^{-1^{\dagger}} \mathbf{B}\right)^{-1} \Rightarrow \\
& {\left[\mathbf{B}^{-1^{\dagger}} \mathbf{B}, \gamma^{\mu}\right]_{-}=0 \text { for all } \mu=\overline{0,3} \text {, respectively, } \mathbf{B}^{\dagger}=} \\
& \lambda \mathbf{B} . \\
\text { (ii) } & \left(\mathbf{B}^{\dagger}\right)^{\dagger}=(\lambda \mathbf{B})^{\dagger}=|\lambda|^{2} \mathbf{B}=\mathbf{B} \Rightarrow|\lambda|=1 .
\end{aligned}
$$

The reasons for the consecrated $\mathbf{B}=\gamma^{0}$ must thus lie in the mathematical foundations of the bispinor space and cannot be collected from the Lagrangian conditions already using $\gamma^{0}$ as adjunctator, other ad hoc conditions (shown in the 
beginning), or representation-specific arguments leading to generalisations not pertinent to the problem.

The representation-independent conditions applicable are

(i) self-adjointness: any $\mathbf{X}^{2}=\mathbf{1}$ has real eigenvalues; hence $\mathbf{X}=\mathbf{X}^{\dagger}$. For example, $\mathbf{X}=\sigma_{i}, \gamma^{0}, \gamma^{5}$, and so forth;

(ii) positive definiteness: for any $\mathbf{X}$, its norm squared $\|\mathbf{X} \psi\|^{2}=\langle\mathbf{X} \psi \mid \mathbf{X} \psi\rangle \geq 0$; hence $\mathbf{B}$ must be such that $\mathbf{X}^{\dagger} \mathbf{X} \geq 0, \forall \mathbf{X}$.

Respectively they are the mathematical translation of understanding the scalar-product. The early arguments of Pauli [3] relied on counting representation-dependent anti-/symmetric matrices. This eludes the above two conditions and leads to one minor misconclusion on $\mathbf{B}$.

\section{Structure of the Scalar Product}

Let $\Gamma=\gamma^{\mu} \gamma^{\nu} \cdots \gamma^{\rho}$; then $\Gamma^{\dagger}=\mathbf{B} \gamma^{\rho} \cdots \gamma^{\nu} \gamma^{\mu} \mathbf{B}^{-1}=\operatorname{sign} \cdot \mathbf{B} \Gamma \mathbf{B}^{-1}$. It is useful thus to define

$$
\bar{\Gamma} \stackrel{\text { def }}{=} \mathbf{B}^{-1} \Gamma^{\dagger} \mathbf{B}
$$

with the trivial action of complex conjugation on scalars and a generalisation of this on operators as follows:

(i) $\overline{\mathrm{XY}}=\overline{\mathbf{Y X}}$,

(ii) $\overline{\mathbf{X}^{-1}}=\overline{\mathbf{X}}^{-1}$,

(iii) $\overline{\mathbf{R}+i \mathbf{C}}=\mathbf{R}-i \mathbf{C}$, for $\mathbf{R}$ and $\mathbf{C}$ "standard", that is, $\overline{\mathbf{R}}=\mathbf{R}$ and $\overline{\mathbf{C}}=\mathbf{C}$;

(iv) $\overline{\bar{\Gamma}} \Gamma=\bar{\Gamma} \Gamma$; that is, $\bar{\Gamma} \Gamma$ is standard;

(v) $\overline{\lambda \mathbf{X}+\mu \mathbf{Y}}=\overline{\lambda \mathbf{X}}+\bar{\mu} \overline{\mathbf{Y}}$;

(vi) if $\mathbf{R}+i \mathbf{C}=0$; then both $\mathbf{R}$ and $\mathbf{C}$ are zero since $\overline{0}=$ $\mathbf{R}-i \mathbf{C}=0$.

With this notation, it is easier to understand the action of $\mathbf{B}$ as follows:

$$
\Gamma^{\dagger}=\mathbf{B} \bar{\Gamma} \mathbf{B}^{-1}
$$

and to further discover the adjunctator: $\mathbf{B}^{\dagger}=\lambda \mathbf{B}=\mathbf{B} \overline{\mathbf{B}} \mathbf{B}^{-1} \Rightarrow$ $\overline{\mathbf{B}}=\lambda \mathbf{B}$. The operator $\overline{\mathbf{B}}$ is comprised of a standard and antistandard part: $\mathbf{B}=\mathbf{R}+i \mathbf{C}$, where both $\mathbf{R}$ and $\mathbf{C}$ are standard. Therefore $\overline{\mathbf{B}}=\lambda \mathbf{B}$ translates to $\mathbf{R}-i \mathbf{C}=\lambda \mathbf{R}+i \lambda \mathbf{C}$, which takes into account that $\lambda=\lambda_{R}+i \lambda_{C}$ and also in view of property (vi) becomes.

$$
\left(\begin{array}{cc}
\lambda_{R}-1 & -\lambda_{C} \\
\lambda_{C} & \lambda_{R}+1
\end{array}\right)\left(\begin{array}{l}
\mathbf{R} \\
\mathbf{C}
\end{array}\right)=0
$$

Respectively, the determinant must vanish (which for $|\lambda|=1$, the case at hand, actually does). The condition from (5) is that $\mathbf{R} \sim \mathbf{C}$, or $\mathbf{B}=z \mathbf{R}$ where $z$ is a unit complex number relevant only for aesthetical purposes as follows:

$$
\Gamma^{\dagger}=(z \mathbf{B}) \bar{\Gamma}(z \mathbf{B})^{-1}=\mathbf{B} \bar{\Gamma} \mathbf{B}^{-1} .
$$

The only effect of $z$ is that of choice: with $z=1, \mathbf{B}^{\dagger}=\mathbf{B}$, with $z=i, \mathbf{B}^{\dagger}=-\mathbf{B}$, with $z=e^{i \theta / 2}, \mathbf{B}^{\dagger}=e^{i \theta} \mathbf{B}$-therefore $z$ can be chosen 1 , hence $\mathbf{B}$ standard and $\mathbf{B}^{\dagger}=\mathbf{B}$. The property of significance is that the adjunctator is, up to a unit complex multiplier, standard. The arguments in [3] of counting symmetric and antisymmetric matrices, though reaching the same conclusion, forbid $\mathbf{B}^{\dagger}=-\mathbf{B}$ which, as shown above, could have served equally well.

\section{Self-Adjoint Operators}

The simplest proof from this point on is by looking at the generators of the Dirac algebra- $(\vec{\sigma}, \vec{g})$, where $\vec{\sigma}=\gamma^{0} \vec{\gamma} \gamma^{5}$ and $\vec{g}=\left(\gamma^{5},-i \gamma^{0} \gamma^{5}, \gamma^{0}\right)$; the two sets defining two isomorphic halves of the space [8] are shown as follows:

$$
\begin{aligned}
& \vec{\sigma} \times \vec{\sigma}=2 i \vec{\sigma}, \quad \text { with } \sigma_{i}^{2}=\mathbf{1}, \\
& \vec{g} \times \vec{g}=2 i \vec{g}, \quad \text { with } g_{i}^{2}=\mathbf{1} .
\end{aligned}
$$

The two halves of the space admit various other representations, such as $\left(1-\gamma^{5}\right) \vec{\sigma} / 2$ and $\left(1+\gamma^{5}\right) \vec{\sigma} / 2$; however, the one presented is the most convenient for the task at hand. The endomorphism between the two halves is $\mathbf{S}=\mathbf{1}+\vec{\sigma} \vec{g}=$ $\mathbf{1}+\gamma^{0} \gamma^{1}-i \gamma^{2}+i \gamma^{0} \gamma^{1} \gamma^{2}$.

The two halves seem identical; however from the adjunctation point of view they differ as follows:

$$
\begin{aligned}
& \overline{\left(\sigma_{1}, \sigma_{2}, \sigma_{3}\right)}=\left(\sigma_{1}, \sigma_{2}, \sigma_{3}\right), \\
& \overline{\left(g_{1}, g_{2}, g_{3}\right)}=\left(-g_{1},-g_{2}, g_{3}\right) .
\end{aligned}
$$

Encouraged by the fact that $\sigma_{i}^{2}=\mathbf{1}$ and $g_{i}^{2}=1$, we explore under what conditions $\vec{\sigma}^{\dagger}=\vec{\sigma}$ and $\vec{g}^{\dagger}=\vec{g}$.

For the first half of the space, we seek an adjunctator in the form of $B=\lambda+\vec{a} \vec{\sigma}$, where $[\lambda, \vec{\sigma}]_{-}=0$ and $[\vec{a}, \vec{\sigma}]_{-}=0$. With some loss of generality, we let $[\lambda, \vec{a}]_{-}=0$ and $\vec{a} \times \vec{a}=0$, for the sake of exemplifying the mechanisms in place. Under this adjunctator;

$$
\vec{\sigma}^{\dagger}=\vec{\sigma}+\frac{2}{\lambda^{2}-\vec{a}^{2}}\left[\vec{a}^{2} \vec{\sigma}_{\perp}-\lambda \vec{a} \times \vec{\sigma}\right]
$$

For $X=\vec{b} \vec{\sigma}$, in order that $X^{\dagger} X \geq 0$, it is evident that $\vec{a}=0$, leaving $\lambda=$ scalar $+\operatorname{LIN}\{\vec{g}\}$.

For the second half of the space, similar arguments follow, only that in this case the $\bar{g}_{i}$ 's are not similar to the $\bar{\sigma}_{i}$ 's. Further restricting generality, assume $\vec{a}=a \vec{e}_{3}$; then

$$
\vec{g}^{\dagger}=\vec{g}-\frac{2}{\lambda^{2}-\vec{a}^{2}}\left[\lambda^{2} \vec{g}_{\perp}-\lambda \vec{a} \times \vec{g}\right]
$$

The role of $\vec{a}=0$ in the first half transfers here to $\lambda=0$.

It can be seen that $B=g_{3}$ performs $\vec{\sigma}^{\dagger}=\vec{\sigma}$ and $\vec{g}^{\dagger}=\vec{g}$, which for any operator in the space satisfies $X^{\dagger} X \geq 0$.

Conversely, without proof, if $\vec{\sigma}^{\dagger}=\vec{\sigma}$ and $\vec{g}^{\dagger}=\vec{g}$, then any operator in the space satisfies $X^{\dagger} X \geq 0$. 
Let $\widetilde{X}=\gamma^{0} \bar{X} \gamma^{0}$ be an operation with the same properties as $\overline{\mathbf{X}}$, mainly just complex conjugating the coefficients in a sum of operators. If $\mathbf{A}=\mathbf{B} \gamma^{0}$, then $X^{\dagger}=\mathbf{A} \widetilde{X} \mathbf{A}^{-1}$ and

$$
\begin{aligned}
& \sigma_{i}^{\dagger}=\mathbf{A} \widetilde{\sigma}_{i} \mathbf{A}^{-1}=\mathbf{A} \sigma_{i} \mathbf{A}^{-1}=\sigma_{i}, \\
& g_{i}^{\dagger}=\mathbf{A} \widetilde{g}_{i} \mathbf{A}^{-1}=\mathbf{A} g_{i} \mathbf{A}^{-1}=g_{i},
\end{aligned}
$$

as assumed, hinted by the fact that $\sigma_{i}$ and $g_{i}$ squared are unity and have real eigenvalues. This implies that

$$
\begin{array}{ll}
{\left[\mathbf{A}, \sigma_{i}\right]_{-}=0,} & \forall i=\overline{1,3}, \\
{\left[\mathbf{A}, g_{i}\right]_{-}=0,} & \forall i=\overline{1,3},
\end{array}
$$

and in turn

$$
\begin{aligned}
& \mathbf{A} \gamma^{0} \gamma^{i} \gamma^{5}=\gamma^{0} \gamma^{i} \gamma^{5} A, \\
& \mathbf{A} \gamma^{0}=\gamma^{0} \mathbf{A}, \\
& \mathbf{A} \gamma^{5}=\gamma^{5} \mathbf{A} .
\end{aligned}
$$

Respectively, both $\left[\mathbf{A}, \gamma^{i}\right]_{-}=0$ and $\left[\mathbf{A}, \gamma^{0}\right]_{-}=0$; that is, $\left[\mathbf{A}, \gamma^{\mu}\right]_{-}=0, \forall \mu=\overline{0,1}$, or $\mathbf{A}=\mathbf{1}$.

All these exemplifications point to the asymmetry-with respect to adjunctation - of the two halves of the space and to the fact that $g_{3}=\gamma^{0}$ may be the Bargmann-Pauli adjunctator.

\section{Positive Definiteness of $X^{\dagger} X$}

A more involved argument is that $\mathbf{B}$ should be such that $X^{\dagger} X$ is positive definite for any $X$; that is: $\mathbf{A} \widetilde{X} \mathbf{A}^{-1} X \geq 0$, which is the matrix version of understanding the scalar product. Since $(\vec{\sigma}, \vec{g})$ have the same properties, and the arbitrariness of $X$ imposes equal constraints on both, that is, any property holding for $X(\vec{\sigma}, \vec{g})$ must also hold for $X(\vec{g}, \vec{\sigma})$, it follows that A is symmetric in $(\vec{\sigma}, \vec{g})$ as follows:

$$
\mathbf{A}=b \cdot \mathbf{1}+\vec{a}(\vec{\sigma}+\vec{g})+M_{i j}\left(\sigma_{i} g_{j}+\sigma_{j} g_{i}\right) .
$$

In the above, $\vec{a}$ and $M_{i j}$ are unjustifiable anisotropies; hence $\vec{a}=0$ and $M_{i j}=\beta \delta_{i, j}$; therefore $\mathbf{A}$ reduces to $\mathbf{A}=\alpha \cdot \mathbf{1}+\beta \mathbf{S}$, with $\mathbf{A}^{-1}=(\alpha-\beta \mathbf{S}) /\left(\alpha^{2}-4 \beta^{2}\right)$, where $\mathbf{S}^{\mathbf{2}}=4 \cdot \mathbf{1}$. The $\mathbf{A} \widetilde{X} \mathbf{A}^{-1} X \geq 0$ inequality becomes

$$
\begin{aligned}
\mathbf{A} \widetilde{X} \mathbf{A}^{-1} X= & \left(\alpha^{2}-4 \beta^{2}\right)^{-1} \\
& \times\left[\alpha^{2} \widetilde{X}_{\sigma}-\beta^{2} \widetilde{X}_{g}+\alpha \beta \mathbf{S}\left(\widetilde{X}_{\sigma}-\widetilde{X}_{g}\right)\right] X_{\sigma} \geq 0,
\end{aligned}
$$

where $X_{\sigma}=X(\vec{\sigma}, \vec{g})$ and $X_{g}=X(\vec{g}, \vec{\sigma})$, $\mathbf{S}$ playing the gobetween role: $\mathbf{S} X_{\sigma}=X_{g} \mathbf{S}$ and $X_{\sigma} \mathbf{S}=\mathbf{S} X_{g}$. Let $X_{\sigma}=X_{g}=$ $\lambda+\mu \mathbf{S}$; then (15) (using $\widetilde{\mathbf{S}}=\mathbf{S}$ ) becomes

$$
\begin{aligned}
\mathbf{A} \widetilde{X} \mathbf{A}^{-1} X= & \left(\alpha^{2}-4 \beta^{2}\right)^{-1} \\
& \times\left[\bar{\lambda}\left(\alpha^{2}-\beta^{2}\right)+\bar{\mu}\left(\alpha^{2}+\beta^{2}\right)+8 \bar{\mu} \alpha \beta\right] \\
& \times(\lambda+\mu \mathbf{S}) \geq 0 .
\end{aligned}
$$

Expanding the above,

$$
\begin{aligned}
\mathbf{A} \widetilde{X} \mathbf{A}^{-1} X=\left(\alpha^{2}-4 \beta^{2}\right)^{-1} & \\
\times[ & (\lambda \bar{\mu}+\bar{\lambda} \mu) \alpha^{2} \mathbf{S}+(\lambda \bar{\mu}-\bar{\lambda} \mu) \beta^{2} \mathbf{S} \\
& +|\lambda|^{2}\left(\alpha^{2}-\beta^{2}\right)+4(\lambda \bar{\mu}+\bar{\lambda} \mu) \alpha \beta \\
& +8 \alpha \beta|\mu|^{2} \mathbf{S}+4|\mu|^{2}\left(\alpha^{2}+\beta^{2}\right) \\
& +4(\lambda \bar{\mu}-\bar{\lambda} \mu) \alpha \beta] \geq 0,
\end{aligned}
$$

where the imaginary boxed terms disappear for $\beta=0$; hence $\mathrm{A} \sim \mathbf{1}$ and $\mathrm{B}=\gamma^{0}$ (qed). Note that the expectation values of $\mathbf{S}=\mathbf{1}+\vec{\sigma} \cdot \vec{g}$ can be $1 / 4$ and $7 / 4$, stemming from the product of two independent $1 / 2$ angular momenta.

In general form (without the anisotropy argument), the proof ends annihilating all $\vec{a}$ and nondiagonal $M_{i j}$ coefficients for the same reason above: the existence of imaginary terms in a relation that needs to be real and positive.

The unicity issue has been addressed by direct construction, although proof in this respect can be found in $[4,5]$.

Another aspect of interest is the possibility of using the $\overline{\mathrm{bar}}$ in place of (adjunctation) ${ }^{\dagger}$ on the helicity-positive subspace as follows:

$$
(\psi \mid \phi) \stackrel{\text { def }}{=}\langle\mathbf{C} \psi \mid \mathbf{C} \phi\rangle .
$$

In this case, $\mathbf{B}=\mathbf{C} \overline{\mathbf{C}}>0$, the $\mathbf{C}$ operator taking the in-between bearing role of $\gamma^{0}$. Evidently, any operator satisfying $\overline{\mathbf{S}} \mathbf{S}=\mathbf{1}$ invaries the new scalar product of $(\psi \mid \phi)$. The particularity of this context is that any $\mathbf{C}$ satisfies the scalar product and norm conditions (i.e., absolutely arbitrary scalar product), leading from the physics point of view to arbitrary many Lorentz invariant conserved currents.

\section{Dirac Currents}

Another interesting aspect is that two Lorentz invariant quantities which include charge density $\langle\rho\rangle=|\psi(x)|^{2}$ can be defined: $\rho \mathbf{B} \gamma^{\mu}$ and $\rho \mathbf{B} \gamma^{\mu} \gamma^{5}$. Of these two, only the former is conserved: $\partial_{\mu}\left\langle\rho \mathbf{B} \gamma^{\mu}\right\rangle_{\psi}=0$.

It is clear that if the adjunctator $\mathbf{B}$ is not unique, more than one such conserved Lorentz invariant quantity can be defined. The transformation properties under space-, time-, and space-time inversion for $j^{\mu}=\left\langle\rho \gamma^{0} \gamma^{\mu}\right\rangle_{\psi}$ are

$$
\begin{aligned}
& j^{\mu}(\psi) \stackrel{\mathbf{P}}{\longrightarrow}-j^{\widehat{\mu}}(\psi), \\
& j^{\mu}(\psi) \stackrel{\mathrm{T}}{\longrightarrow}-j^{\widehat{\mu}}(\psi), \\
& j^{\mu}(\psi) \stackrel{\mathbf{P T}}{\longrightarrow} j^{\mu}(\psi),
\end{aligned}
$$

where $\mathbf{U}_{P}^{\dagger}=-\mathbf{U}_{P}, \mathbf{U}_{T}^{\dagger}=-\mathbf{U}_{T}$ and $\mathbf{U}_{P T}^{\dagger}=\mathbf{U}_{P T}$ were evidently included. The notation $a^{\widehat{\mu}}$ refers to $g^{\mu \nu} a_{\nu}$. Above, $j^{\mu}$ is not an operator, but rather the product: $j^{\mu}=\langle\rho\rangle_{\psi} \cdot\left\langle\mathbf{v}^{\mu}\right\rangle_{\psi}$. Its 
transformation properties seem to differ from textbook electrodynamics by the minus sign. This comes from the fact that charge density is better defined as a pseudoscalar under both space- and time-inversion [9-12]. Incidentally, this also solves the problem of not finding a representation-independent charge-conjugation operator $\mathbf{C}$, since time reversal does this automatically for all currents, without the need of a specific charge-conjugation operator for each current in its own space.

\section{Conclusion}

It has been shown in representation-independent form that the adjunctator for the Dirac space is the $\gamma^{0}$ matrix. This is an important result, as the adjunctator enters the definition of the Dirac current and multiple adjunctators would imply the possibility of defining multiple currents, which, incidentally, is possible for the positive helicity subspace.

As the adjunctator plays also the role of parity transformation, its unicity is essential to the theory. Furthermore, together with time inversion, it defines complete coordinate inversion, which reverses all currents-without the need for a charge-conjugation operator. The latter is impossible to define in representation-independent form, which in itself is a statement over why it does not exist and why it is important to have representation-independent results.

\section{Acknowledgments}

This work was supported by a Grant of the Romanian National Authority for Scientific Research, CNCSUEFISCDI, Project no. PN-II-ID-PCE-2011-3-0323.

\section{References}

[1] W. Pauli, "Über die Formulierung der Naturgesetze mit fünf homogenen Koordinaten. Teil II: Die Diracschen Gleichungen für die Materiewellen," Annalen Der Physik, vol. 410, no. 4, pp. 337-372, 1933.

[2] W. Pauli, "On the formulation of the laws of Nature with five homogeneous components. Part II: the Dirac equation for matter waves," Annals of Physics, vol. 18, pp. 337-372, 1933.

[3] W. Pauli, "Contributions mathématiques à la théorie des matrices de Dirac," Annales de L'Institut Henri Poincaré, vol. 6, pp. 109-136, 1936.

[4] M. Arminjon and F. Reifler, "Four-vector versus four-scalar representation of the Dirac wave function," International Journal of Geometric Methods in Modern Physics, vol. 9, no. 4, Article ID 1250026, 2012.

[5] M. Arminjon and F. Reifler, "Dirac equation: representation independence and tensor transformation," Brazilian Journal of Physics, vol. 38, no. 2, pp. 248-258, 2008.

[6] M. Arminjon, "A simpler solution of the non-uniqueness problem of the covariant Dirac theory," International Journal of Geometric Methods in Modern Physics, vol. 10, no. 7, Article ID 1350027, 2013.

[7] M. Arminjon and F. Reifler, "A non-uniqueness problem of the Dirac theory in a curved spacetime," Annalen Der Physik, vol. 523, no. 7, pp. 531-551, 2011.
[8] P. Caban, J. Rembielinski, and M. Wlodarczyk, "Spin operator in the Dirac theory," Physical Review A, vol. 88, no. 2, Article ID 022119, 2013.

[9] R. M. Kiehn, "Charge is a pseudo scalar," http://www22.pair .com/csdc/pdf/ptpost.pdf.

[10] J. Schwinger, "Quantum electrodynamics. I: a covariant formulation," Physical Review, vol. 74, no. 10, pp. 1439-1461, 1948.

[11] R. P. Feynman, "The theory of positrons," Physical Review, vol. 76, no. 6, pp. 749-759, 1949.

[12] M. Dima, "Representation independent CPT transformations," Internal Note IFIN-PUB-0487, Institute for Nuclear Physics and Engineering, 2012. 

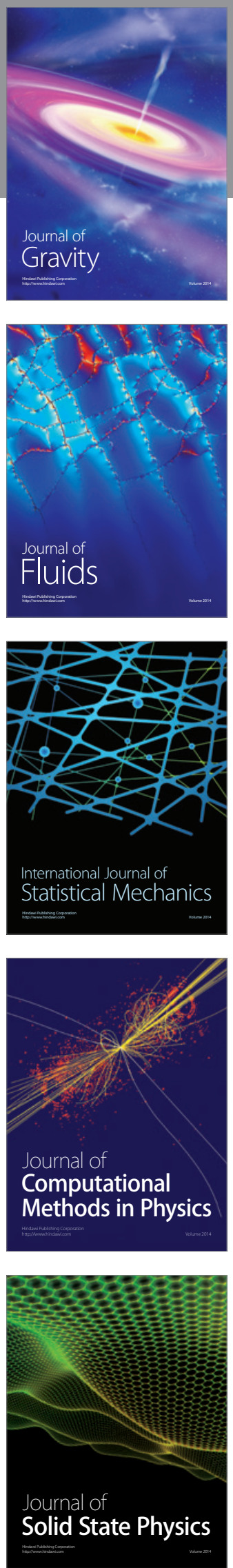

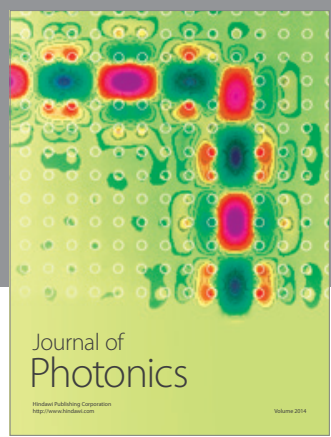

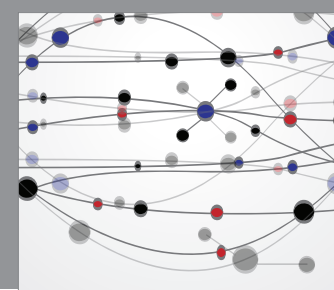

The Scientific World Journal

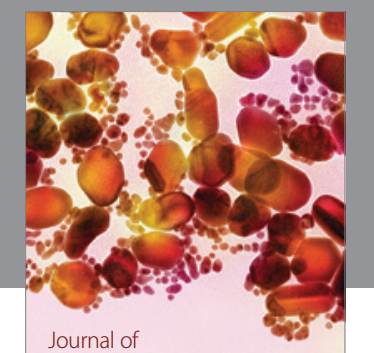

Soft Matter
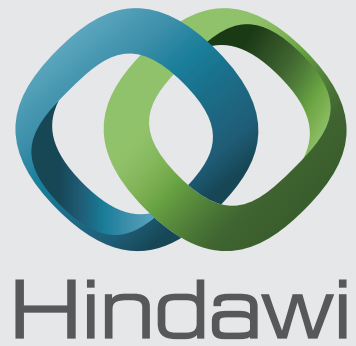

Submit your manuscripts at

http://www.hindawi.com
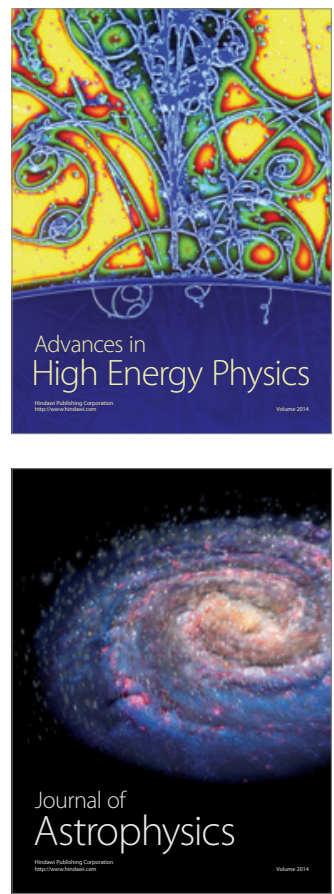
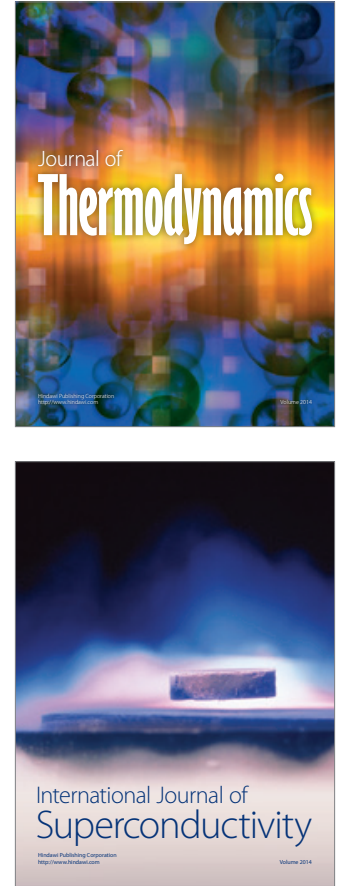
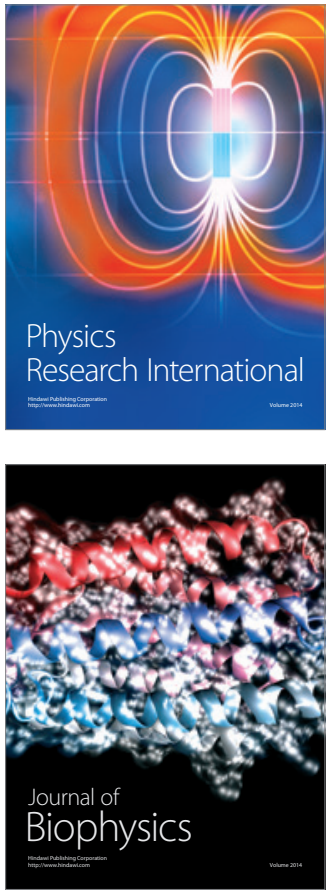
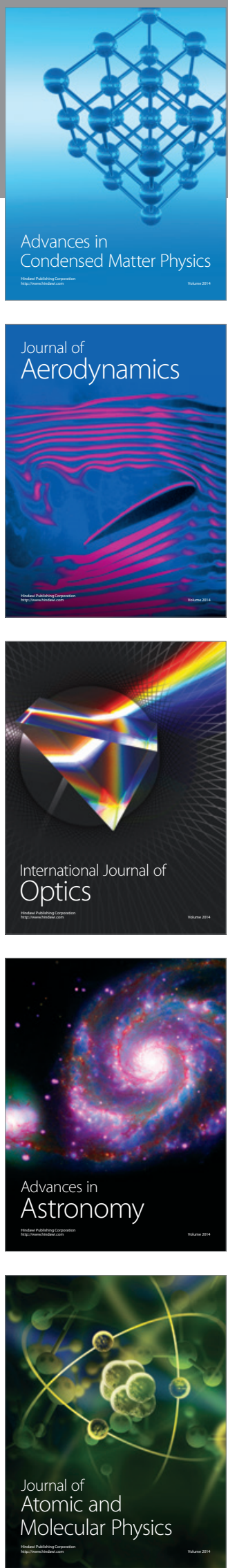